\title{
The Dilemma of Ureterovesical Junction Obstruction
}

\author{
Seyed Mohammadreza Rabani, ${ }^{1, *}$ and Ali Mousavizadeh ${ }^{2}$ \\ ${ }^{1}$ Associate Professor of Urology and Renal Transplantation, Beheshti Teaching Hospital, Yasuj University of Medical Sciences, Yusuj, Iran \\ ${ }^{2}$ Assistant Professor of Epidemiology, Social Determinants of Health Research Center, Yasuj University of Medical Sciences, Yusuj, Iran \\ "Corresponding author: Seyed Mohammadreza Rabani, Associate Professor of Urology and Renal Transplantation, Beheshti Teaching Hospital, Yasuj University of Medical \\ Sciences, Yusuj, Iran. Tel: +98-9177411389, E-mail: smrrabani@yahoo.com
}

Received 2017 April 17; Revised 2017 July 01; Accepted 2017 August 20.

\begin{abstract}
Background: Ureterovesical junction obstruction (UVJO) is the result of an anatomic or a functional abnormality in the distal segment of the ureter. There are many types of UVJO, different in their cause, presentation, imaging characters, and prognosis.

Objectives: The aim of this study was to discuss the postoperative natural course and prognosis of patients undergoing standard approaches on the management of primary UVJO by one surgeon in a 10-year period starting from 2004.

Methods: From January 2004 to October 2013, 64 patients who underwent ureteral tapering and stenting ureteroneocystostomy for complicated primary UVJO participated in the study.

Results: The mean age of patients was 26 years (range: 11 months to 73 years). The mean hospital stay was 5 days (range: 4 to 7 days). The post-operative time was between 1 and 2 hours. Double J stent was left in place for 4 weeks. Post-operative follow-up was at least one year. No major complication was encountered, instead, a decrease in symptoms in all patients and an increase in renal function in 40 out of 64 (62.5\%) patients were observed in the one-year follow-up of the diethylenetriaminepentaacetic acid (DTPA) renal scanning. Only 3 patients had fever as a postoperative minor complication.

Conclusions: Decision making in the treatment of UVJO is somewhat a dilemma. This condition is occasionally asymptomatic and uneventful, and may be detected accidentally during an unrelated work-up; nonetheless, it may also cause serious life threatening complications. Surgical management in complicated cases may be a safe and viable treatment option both in children and in adults.
\end{abstract}

Keywords: Congenital Abnormalities, Ureteral Obstruction, Hydroureteronephrosis

\section{Background}

UVJO is the result of an anatomic or a functional abnormality in the distal portion of the ureters. Gil Vernet described the normal anatomy of the ureterovesical junction, and explained 3 distinguished segments at this part of the ureter: juxta-vesical, intramural, and sub mucosal segments, associated by Waldeyer's sheath and pre-vesical sphincter (1). After ureteropelvic junction obstruction (UPJO), UVJO is the second most common cause of hydronephrosis in newborns, accounting for approximately $20 \%$ of cases, with an estimated incidence of 36 per 100000 live births (2). There are many types of UVJOs, with different causes, presentations, imaging characters, and prognosis. Decision making in this condition also depends on the types of UVJO and its related characteristics. Although the true pathogenesis of primary UVJO is uncertain, it appears that it is usually a result of an abnormality or delay in the development of muscles in the distal fetal ureter at 20th week of gestation. Historically, different types of open surgery including anti-refluxing or simple ureteral re-implantation with or without ureteral tapering, laparoscopic or robotic surgery, and endoscopic management have been used for the treatment of primary
UVJO. Nephrectomy may be rarely suggested in symptomatic patients with a nonfunctional kidney.

Decision making in the treatment of UVJO is somewhat a dilemma. This condition is occasionally asymptomatic and uneventful and may be detected accidentally during an unrelated work up, but it may also cause serious life threatening complications. There are controversies on the approach toward primary UVJO cases in clinical practices, especially in patients that refer to a clinic by flank pain without any serious complicated event. While a number of experts consider conservative approaches in dealing with the asymptomatic disease, some experts prefer surgery. The main claim of conservative expert opinions is insufficiency of surgery, and postoperative complications for the patient. There are many types of complications occurring after UVJO operation. An important role of the physician is to conduct a good and long-term follow-up for these patients after treatment.

Complications such as urinary extravasation, collection, infection, and ureteral stricture may be encountered. The follow-up and reporting of probable complications in long term is not only a task for physician to ensure about the efficacy of intervention and satisfaction of patients, but also important to evaluate and compare methods used by 
different subspecialists in other settings.

\section{Objectives}

The aim of this study was to discuss the postoperative natural course and prognosis in patients undergoing standard approaches for the management of primary UVJO by one surgeon in a 10-year period from 2004.

\section{Methods}

In the present longitudinal follow-up design (nonconcurrent single cohort study), among a group of 531 patients with primary UVJO, 64 patients $(12.05 \%$ ) who underwent ureteral tapering and stenting ureteroneocystostomy, from January 2004 to October 2013, were selected to participate in the study.

In order to obtain a definite diagnosis and to have differential diagnosis between different types of UVJO, after careful history taking and physical examination, ultrasonography, DTPA (diethylenetriaminepentaacetate) scanning, IVP, and voiding cystourethrography (VCUG) were used. Patients were selected for the study if they had complicated primary obstructive mega ureter with acute or recurrent urinary tract infection, stone obstruction, and/or renal function deterioration as inclusion criteria. Exclusion criteria were asymptomatic primary UVJO or other types of UVJO. Moreover, informed consent was taken from the patients or their parents.

One surgical approach was used to manage the patients through a Gibson incision entering the retroperitoneum. The hallmark for finding the ureter was the site of iliac vessels bifurcation where the ureter is easily identified and dissected downward. The ipsilateral umbilical artery was divided and ligated by 3 - 0 silk to prevent further kinking on the ureter. According to the patient's age or size of the ureter, ureteral tapering was done around an 8 or $10 \mathrm{of}$ catheter to create a suitable sub mucosal tunnel in cases of antirefluxing plan. In a group of adult patients, modified lich Gregoir procedure was done. Furthermore, in cases of ureteral stone(s), the stones were removed from the ureteral longitudinal incision. Vicryl 6 - 0 was used for ureterorrhaphy in a continuous manner and reinforced with interrupted 5 - 0 vicryl in separated sutures. Finally, the catheter was changed to a $3 \mathrm{f}, 4.8 \mathrm{f}$, or $6 \mathrm{f}$ double $\mathrm{J}$ stent (according to the size of the ureter and the age of the patient), that was left in place for a period of 4 weeks, and also a drain was placed for a period of 5 to 7 days. Furthermore, all of the patient's symptoms and signs were recorded in an open-ended questionnaire based on standard approaches during the whole study period. All pa- tients were discharged by order of a third generation antibiotic and NSAID drug for 7 days. The follow-up plan was one week (for removing sutures), 4 weeks (for removing the stent), 3 months, 6 months, and 12 months (check-up by UA, UC, kidney-pelvis sonography, and renal DTPA scanning).

Ureteral tapering and antireflux ureteral reimplantation were done in 49 patients; modified Lich Gregoir procedure was done in 15 patients; and double J stent was inserted in both groups. The SPSS ver. 11 software was used for descriptive analysis and graphing the results.

\section{Results}

64 eligible patients participated in the present study. The mean age and standard deviation was $25.45 \pm 14.9$ years (Range: 11 months to 73 years). 16 patients were in the pediatric age group $(<16$ years) and the rest were adults. Male to female ratio was 1.46 (38/26). Renal stone disease in 24 patients, renal function deterioration in 13 patients, urinary tract infection in 9 patients, and pain in 18 patients were key points of patients' attendance and reasons for surgical interventions. The operation time range was between 1 and 2 hours. The mean hospital stay was 5 days (range: 4 to 7 days). Double J stent was left in place for 4 weeks. The post-operative follow-up lasted at least one year. No major complication was encountered; instead, decreasing symptoms in all patients and increasing renal function in 40 out of 64 patients (62.5\%) were observed in the one-year follow-up of the diethylenetriaminepentaacetic acid (DTPA) renal scanning. Only 3 patients had fever as a post-operative minor complication. Table 1 shows the basic characteristics of the patients.

There were no important and considerable complications or recurrent symptoms in any patient after a one-year follow-up. Three patients reported fever in early postoperative periods.

Based on binomial logistic regression analysis, no marked association was observed between predictor variables such as sex, type of compliant, or DTPA finding and post-operative complications.

The spectrum of patients in the present study was very extensive, from a 38 asymptomatic female patient who presented with an unrelated symptom for whom the dye study showed left UVJO (Figure 1), up to an 81-year-old man who presented with pyonephrosis (Figures 2 and 3). In this patient, with giant ureteral stone and pyonephrosis, ureterolithotomy and ureterostomy were conducted in a secondary intervention. 
Table 1. Qualitative Frequency of Patients Enrolled in the Study

\begin{tabular}{|c|c|c|}
\hline Variable Level & No. (\%) & Cumulative Percent \\
\hline \multicolumn{3}{|l|}{ Sex } \\
\hline Female & $26(40.6)$ & 40.6 \\
\hline Male & $38(59.4)$ & 100.0 \\
\hline Total & $64(100.0)$ & \\
\hline \multicolumn{3}{|l|}{ Main findings at evaluation } \\
\hline Recurrent urinary tract infection & $8(12.5)$ & 12.5 \\
\hline Renal function deterioration & $13(20.3)$ & 32.8 \\
\hline Flank pain & $18(28.1)$ & 60.9 \\
\hline Renal stone & $25(39.1)$ & 100 \\
\hline Total & $64(100)$ & \\
\hline \multicolumn{3}{|l|}{ Operation type } \\
\hline Modified lich procedure & $15(23.4)$ & 23.4 \\
\hline Tapering/antireflux & $49(76.6)$ & 100 \\
\hline Total & $64(100)$ & \\
\hline \multicolumn{3}{|l|}{ DTPA scanning } \\
\hline Nonfunctional & $1(1.6)$ & 1.6 \\
\hline Functional & $63(98.4)$ & 98.4 \\
\hline Total & $64(100.0)$ & \\
\hline \multicolumn{3}{|l|}{ Complications } \\
\hline Fever & $3(4.7)$ & 4.7 \\
\hline None & $61(95.3)$ & 100.0 \\
\hline Total & $64(100.0)$ & \\
\hline
\end{tabular}

\section{Discussion}

Primary UVJO is a congenital anomaly of the urinary tract, which is often associated with antenatal hydronephrosis. In the study of developmental process, a genetic basis has been suggested for this anomaly (1). The different course of natural history of this entity associated with different imaging characters has caused dilemma in the presentation, differential diagnosis, and decision making for its management. UVJO causes increment in smooth muscle contractility as well as in cholinergic and adrenergic activity in distal ureter of rabbits (3). UVJO may coexist with UPJO and this may cause a problem both in diagnosis and in management (4-6). In this situation, all diagnostic imaging techniques should be employed for defining the correct diagnosis and proper management of both conditions. Although UVJO is among the most common causes of obstructive uropathy, it may coexist rarely with posterior urethral valve (7); nevertheless, this has been a rare condition so far. It may also rarely be associated with juxta vesical bladder diverticulum (8). Luis Botelho and cowork- ers reported a case of transient UVJO (9). Although transient ureteropelvic junction obstruction (UPJO) is a clinically well-known entity, transient UVJO has not been previously reported. Surgical management in complicated cases may be a safe and viable treatment option both in children and in adults.

L. Garcia Aparicio and coworkers studied the efficacy of high-pressure dilation of ureterovesical junction starting from 2008 to treat primary UVJO and suggested it as the first-line treatment, but with unknown long-term followup (10). Considering the pathophysiology of this condition, long-term efficacy of this treatment may be under question.

Rui He and coworkers reported their novel technique in laparoscopic ureteral reimplantation with extracorporeal tapering and suturing and replacing the nippled ureter from the same access port of the laparoscopy (11). They had a 17-month satisfactory follow-up. In spite of minimal invasion, it is a technically complex procedure and needs reestablishment of the pneumoperitoneum. An- 


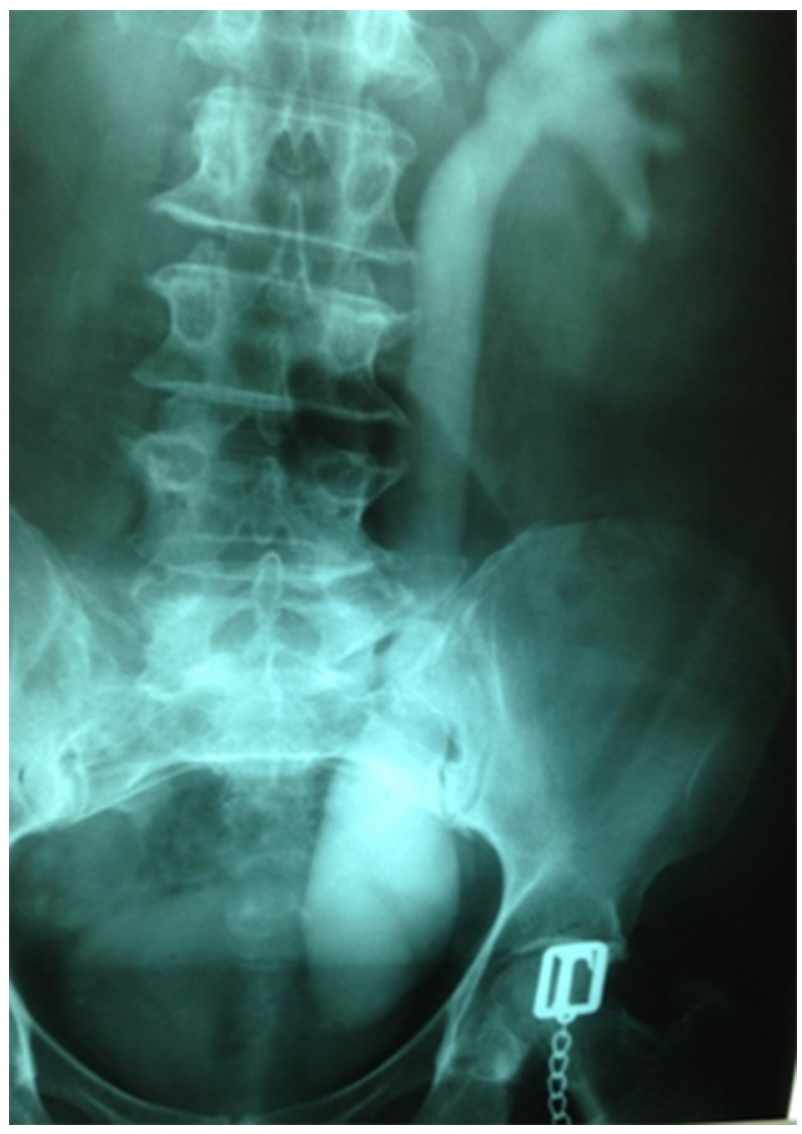

Figure 1. 38-Year-Old Woman with Accidentally Found UVJO

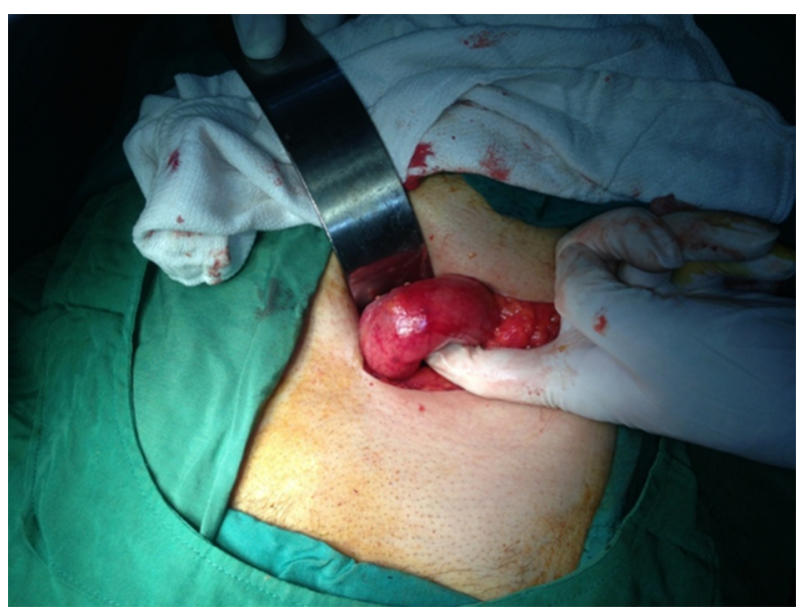

Figure 2. The Dilated Ureter with Stone and Infected Hydroureteronephrosis in a Known Case of Primary Mega Ureter: Urosepsis

other type of laparoscopic intervention was reported by Bondarenko S.(12), who performed a laparoscopic dismem-

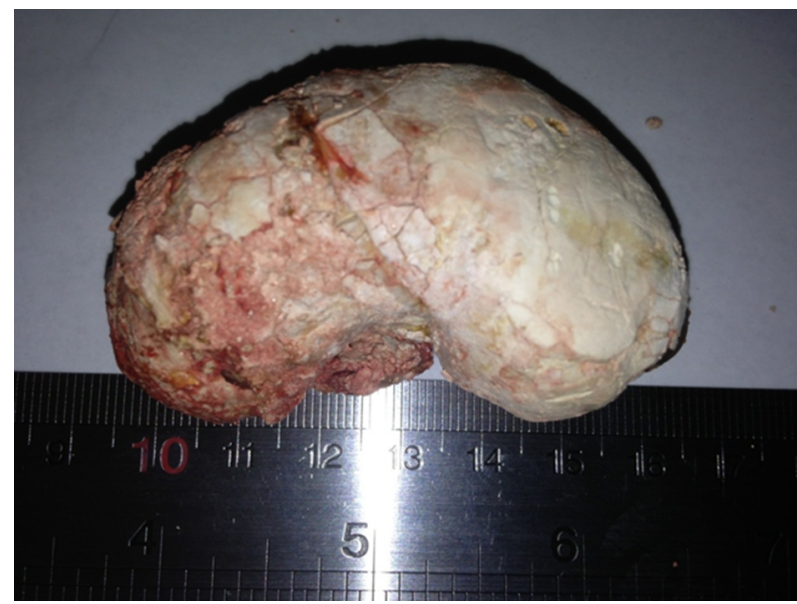

Figure 3. A Giant Ureteral Stone (More Than $6 \mathrm{~cm}$ ) in Distal Part of the Ureter with Infected Hydroureteronephrosis in a Known Case of Primary Mega Ureter.

bered extravesical transverse ureteral reimplantation and suggested this technique as an eventual option in pediatric minimally invasive urologic surgery. However, in this procedure, the mean operative time was about 3 hours and it needed long-term follow-up to evaluate the outcomes.

Herz and coworkers in a study on the efficacy of continuous antibiotic prophylaxis (CAP) in children with antenatally detected hydronephrosis including primary UVJO concluded that CAP might have a significant role in reducing the risk of febrile UTI in these children (13).

To minimize the potential of further injury to the kidney with primary UVJO, Kaefer and coworkers reported a technique in which refluxing ureteral reimplantation as a temporary internal diversion of the obstructed megaureter was suggested, with further definite reimplantation in a second intervention (14). Although this is a simple surgical technique, due to surgical scars, the second intervention may be more difficult to achieve sufficient sub mucosal tunnel.

Miguel arrabal-martin and coworkers introduced an endoscopic oblique meatotomy technique by scissors using an 8.5f ureteroscope in an outpatient basis with 6 weeks DJ insertion (15). They reported an acceptable 3-year follow-up with minimal complication rates. Although this technique has shown low complications, it is a simple and fast procedure conducted only on a small group of patients (18 patients) and has been associated with possible recurrence and vesico ureteral reflux.

Based on our 10-year experience, proper case selection in every age is an important factor. To differentiate a complicated obstructive hydroureteronephrosis from refluxing megaureter, refluxing obstructed ureter, and non- 
refluxing nonobstructed hydroureteronephrosis, proper diagnostic studies were employed and finally, a simple antirefluxing procedure in a well-familiar anatomy through a Gibson incision was applied. In a 10-year follow-up, we obtained acceptable results in eliminating symptoms, reducing hydroureteronephrosis, and increasing renal function.

\subsection{Conclusion}

Decision making in the treatment of UVJO is somewhat a dilemma. This condition is occasionally asymptomatic and uneventful and may be detected accidentally during an unrelated work-up (Figure 1); but it may also cause serious life threatening complications. Surgical management in complicated cases may be a safe and viable treatment option both in children and in adults.

\section{Acknowledgments}

We would like to thank all the patients who cooperated in the process of this study.

\section{Footnote}

Financial Disclosure: We declare that we have no conflict of interest in any terms and conditions related to this investigation.

\section{References}

1. Rasouly HM, Lu W. Lower urinary tract development and disease. Wiley Interdiscip Rev Syst Biol Med. 2013;5(3):307-42. doi: 10.1002/wsbm.1212. [PubMed: 23408557].

2. Stoll C, Alembik Y, Roth MP, Dott B, Sauvage P. Risk factors in internal urinary system malformations. Pediatr Nephrol. 1990;4(4):319-23. [PubMed: 2206898].

3. Yalcin S, Ertunc M, Ardicli B, Kabakus IM, Tas TS, Sara Y, et al. Ureterovesical junction obstruction causes increment in smooth muscle contractility, and cholinergic and adrenergic activity in distal ureter of rabbits. J Pediatr Surg. 2013;48(9):1954-61. doi: 10.1016/j.jpedsurg.2013.01.030. [PubMed: 24074674].
4. Lee YS, Im YJ, Lee H, Kim MJ, Lee MJ, Jung HJ, et al. Coexisting ureteropelvic junction obstruction and ureterovesical junction obstruction: is pyeloplasty always the preferred initial surgery? Urology. 2014;83(2):443-9. doi: 10.1016/j.urology.2013.08.087. [PubMed: 24246322].

5. Moodley P, Demaria J, Lorenzo AJ, Pippi Salle JL, Braga LH. Concurrent ureteropelvic and ureterovesical junction obstruction in children: the value of retrograde pyelography.JPediatr Urol. 2010;6(2):11721. doi: 10.1016/j.jpurol.2009.08.006. [PubMed: 19713154].

6. Ebadi M, Kajbafzadeh AM, Tourchi A, Mousavian AA. Endoureterotomy as the initial management of concurrent ureteropelvic and ureterovesical junction obstruction after failed conservative therapy. Urology. 2013;82(1):214-9. doi: 10.1016/j.urology.2013.02.048. [PubMed: 23601441].

7. Pradhan M, Singh N, Singh AK, Kumari N. Rare association of fetal posterior urethral valve with ureteric stricture. J Prenat Med. 2012;6(1):1-3. [PubMed: 22905302].

8. Torricelli FC, Chueh SC, Shen SJ, Levy DA, Monga M. Hydroureteronephrosis secondary to juxta-uretrovesical junction bladder diverticula. Urology. 2013;82(3):e26-7. doi: 10.1016/j.urology.2013.06.015. [PubMed: 23987182].

9. Botelho L, Rincon JA, Nguyen HT, Bauer SB, Rust D, Estrada CR. Intermittent ureterovesical junction obstruction in children.JPediatr Urol. 2011;7(5):579-81. doi:10.1016/j.jpurol.2011.01.014. [PubMed: 21377934].

10. Garcia-Aparicio L, Rodo J, Krauel L, Palazon P, Martin O, Ribo JM. High pressure balloon dilation of the ureterovesical junction-first line approach to treat primary obstructive megaureter? J Urol. 2012;187(5):1834-8. doi:10.1016/j.juro.2011.12.098. [PubMed: 22425047].

11. He R, Yu W, Li X, Yao L, He Z, Zhou L. Laparoscopic ureteral reimplantation with extracorporeal tailoring and direct nipple ureteroneocystostomy for adult obstructed megaureter: a novel technique. Urology. 2013;82(5):1171-4. doi: 10.1016/j.urology.2013.07.019. [PubMed: 24035035].

12. Bondarenko S. Laparoscopic extravesical transverse ureteral reimplantation in children with obstructive megaureter. J Pediatr Urol. 2013;9(4):437-41. doi:10.1016/j.jpurol.2013.01.001. [PubMed: 23491982].

13. Herz D, Merguerian P, McQuiston L. Continuous antibiotic prophylaxis reduces the risk of febrile UTI in children with asymptomatic antenatal hydronephrosis with either ureteral dilation, high-grade vesicoureteral reflux, or ureterovesical junction obstruction. J Pediatr Urol. 2014;10(4):650-4. doi:10.1016/j.jpurol.2014.06.009. [PubMed: 25155409].

14. Kaefer M, Misseri R, Frank E, Rhee A, Lee SD. Refluxing ureteral reimplantation: a logical method for managing neonatal UVJ obstruction.J Pediatr Urol. 2014;10(5):824-30. doi:10.1016/j.jpurol.2014.01.027. [PubMed: 24850437].

15. Arrabal-Martin M, Zuluaga-Gomez A, Merino-Salas S, Nogueras-Ocana M, Arrabal-Polo MA. Endoscopic treatment of ureterovesical junction obstructive pathology: A description of the oblique meatotomy technique and results. Can Urol Assoc J. 2013;7(11-12):E728-31. doi: 10.5489/cuaj.496. [PubMed: 24282466]. 